\title{
NOTE ON A PAPER BY MAHLER
}

\author{
By
}

\author{
Numiko NisHioKA
}

\section{Introduction.}

Let $\omega$ be a real quadratic irrational number with $0<\omega<1$, and put

$$
F_{\omega}\left(z_{1}, z_{2}\right)=\sum_{h_{1}=1}^{\infty} \sum_{h_{2}=1}^{\left[h_{1} \omega\right]} z_{1}{ }^{h_{1}} z_{2}{ }^{h_{2}} .
$$

The series $F_{\omega}\left(z_{1}, z_{2}\right)$ converges in the domain

$$
\left\{\left|z_{1}\right|<1,\left|z_{1}\right|\left|z_{2}\right|^{\omega}<1\right\} .
$$

Mahler [3] proves that $F_{\omega}\left(\alpha_{1}, \alpha_{2}\right)$ is transcendental for algebraic $\alpha_{1}, \alpha_{2}$ with suitable properties. In the succeeding paper [4], he studies the algebraic independence of the values

$$
\left.\frac{\partial^{k_{1}+k_{2}} F_{\omega}\left(z_{1}, z_{2}\right)}{\partial z_{1}{ }^{k_{1}} \partial z_{2}^{k_{2}}}\right|_{\left(\alpha_{1}, \alpha_{2}\right)}, \quad k_{1} \geqq 0, k_{2} \geqq 0 .
$$

To prove the algebraic independence of the values, he asserts the functions

$$
\frac{\partial^{k_{1}+k_{2}} F_{\omega}\left(z_{1}, z_{2}\right)}{\partial z_{1}{ }^{k} \partial z_{2}{ }^{k_{2}}}, \quad k_{1} \geqq 0, k_{2} \geqq 0,
$$

are algebraically independent over the rational function field $C\left(z_{1}, z_{2}\right)$. But it is pointed out in Kubota [1] and Loxton and van der Poorten [2] that Mahler's criterion for algebraic independence (Satz 1 in [4]) is not correct. Although the correct criterion is given in [1] and [2], it seems that there is no proof of the algebraic independence of the functions (3). Here we will prove the following theorems.

THEOREM 1. The functions (3) are algebraically independent over $\boldsymbol{C}\left(z_{1}, z_{2}\right)$.

Let $\omega$ be expanded in the continued fraction

$$
\omega=\frac{1}{a_{1}+\frac{1}{a_{2}+\cdots}},
$$

Received June 10, 1992, Revised November 5, 1992. 
and

$$
\begin{aligned}
& p_{-1}=0, \quad p_{0}=1, \quad p_{1}=a_{1}, \quad p_{\mu+1}=a_{\mu+1} p_{\mu}+p_{\mu-1}, \\
& q_{-1}=1, \quad q_{0}=0, \quad q_{1}=1, \quad q_{\mu+1}=a_{\mu+1} q_{\mu}+q_{\mu-1} .
\end{aligned}
$$

From Theorem 1 and the main theorem in [4], we obtain the following theorem.

THEOREM 2. Let $\alpha_{1}, \alpha_{2}$ be algebraic numbers satisfying

$$
0<\left|\alpha_{1}\right|<1, \quad 0<\left|\alpha_{1}\right|\left|\alpha_{2}\right|^{\omega}<1, \quad \alpha_{1}{ }^{p} \alpha_{2}{ }^{q_{\mu}} \neq 1 \quad(\mu \geqq 0) .
$$

Then the values (2) are algebraically independent.

COROLlaRY. Let $f(z)=\sum_{h=1}^{\infty}[h \omega] z^{h}$ and $\alpha$ an algebraic number with $0<|\alpha|$ $<1$. Then

are algebraically independent.

$$
f^{(k)}(\boldsymbol{\alpha}), \quad k \geqq 0
$$

\section{Proof of the theorems.}

Define $\omega_{1}, \omega_{2}, \cdots$ by

$$
\omega=\frac{1}{a_{1}+\omega_{1}}, \quad \omega_{1}=\frac{1}{a_{2}+\omega_{2}}, \cdots
$$

Because of the equality (see [3])

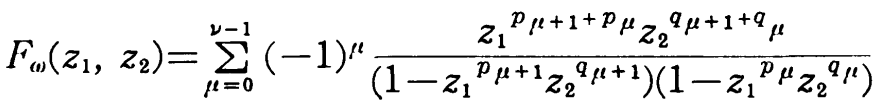

$$
\begin{aligned}
& +(-1)^{\nu} F_{\omega_{\nu}}\left(z_{1}^{p_{\nu}} z_{2}^{q_{\nu}}, z_{1}^{p_{\nu-1}} z_{2}^{q_{\nu-1}}\right) \text {, }
\end{aligned}
$$

we may assume that the continued fraction of $\omega$ is purely periodic. Therefore there exists a natural number $\nu$ such that $\omega=\omega_{\nu}$. We may assume $\nu$ is even. Put

$$
\Omega=\left(\begin{array}{ll}
p_{\nu} & q_{\nu} \\
p_{\nu-1} & q_{\nu-1}
\end{array}\right) \text { and } \Omega\left(z_{1}, z_{2}\right)=\left(z_{1}^{p_{\nu}} z_{2}^{q_{\nu}},{z_{1}}^{p_{\nu-1}} z_{2}^{q_{\nu-1}}\right)
$$

Then we have

$$
F_{\omega}\left(z_{1}, z_{2}\right)=F_{\omega}\left(\Omega\left(z_{1}, z_{2}\right)\right)+b\left(z_{1}, z_{2}\right), \quad b\left(z_{1}, z_{2}\right) \in \boldsymbol{Q}\left(z_{1}, z_{2}\right) .
$$

Let $\rho_{1}=p_{\nu}+p_{\nu-1} \omega, \rho_{2}=q_{\nu-1}-p_{\nu-1} \omega$. Then $\rho_{1}, \rho_{2}$ are the eigen values of the matrix $\Omega$ and

$$
\left(\begin{array}{l}
o_{1}{ }^{(\lambda)} \\
o_{2}(\lambda)
\end{array}\right)=\left(\begin{array}{c}
q_{\nu-1}-\rho_{\lambda} \\
-p_{\nu-1}
\end{array}\right)
$$

is an eigenvector belonging to $\rho_{\lambda}(\lambda=1,2)$. Put 


$$
D_{\lambda}=o_{1}{ }^{(\lambda)} z_{1} \frac{\partial}{\partial z_{1}}+o_{2}{ }^{(\lambda)} z_{2} \frac{\partial}{\partial z_{2}}, \quad \lambda=1,2 .
$$

Then we have $([4], \S 9)$,

$$
\begin{aligned}
& D_{1}{ }^{k_{1}} D_{2}{ }^{k_{2}} f\left(\Omega\left(z_{1}, z_{2}\right)\right) \\
& \quad=\left.\rho_{1}{ }^{k_{1}} \rho_{2}{ }^{k_{2}} D_{1}{ }^{k_{1}} D_{2}{ }^{k_{2}} f\left(z_{1}, z_{2}\right)\right|_{\Omega\left(z_{1}, z_{2}\right)}, k_{1}, k_{2} \geqq 0 .
\end{aligned}
$$

where $f\left(z_{1}, z_{2}\right)$ is any analytic function. By the equality (8), we have

$$
\begin{aligned}
& D_{1}{ }^{k_{1}} D_{2}{ }^{k_{2}} F_{\omega}\left(z_{1}, z_{2}\right) \\
& \quad=\left.\rho_{1}{ }^{k_{1}} \rho_{2}{ }^{k_{2}} D_{1}{ }^{k_{1}} D_{2}{ }^{k_{2}} F_{\omega}\left(z_{1}, z_{2}\right)\right|_{\Omega\left(z_{1}, z_{2}\right)}+D_{1}{ }^{k_{1}} D_{2}{ }^{k_{2}} b\left(z_{1}, z_{2}\right) .
\end{aligned}
$$

We shall prove that the functions

$$
D_{1}^{k_{1}} D_{2}^{k_{2}} F_{\omega}\left(z_{1}, z_{2}\right), \quad k_{1}, k_{2} \geqq 0,
$$

are algebraically independent over $C\left(z_{1}, z_{2}\right)$, from which Theorem 1 and Theorem 2 follow, since $\operatorname{det}\left(\begin{array}{cc}o_{1}{ }^{(1)} & o_{1}{ }^{(2)} \\ o_{2}{ }^{(1)} & o_{2}{ }^{(2)}\end{array}\right) \neq 0$. The proof is by contradiction. We assume the functions (10) were algebraically dependent over $\boldsymbol{C}\left(z_{1}, z_{2}\right)$. Let $K=\boldsymbol{Q}(\boldsymbol{\omega})$. Since the Taylor coefficients of the functions (10) are in $K$, the functions are algebraically dependent over $K\left(z_{1}, z_{2}\right)$. By Corollary 9 in [1], the functions (10)] are $K$-linearly dependent $\bmod K\left(z_{1}, z_{2}\right)$. (Kubota [1] states the corollary over the field $\boldsymbol{C}$, but it is easily checked that the above statement is also valid.) Therefore the functions

$$
F^{\left(k_{1}, k_{2}\right)}\left(z_{1}, z_{2}\right)=\left(z_{1} \frac{\partial}{\partial z_{1}}\right)^{k_{1}}\left(z_{2} \frac{\partial}{\partial z_{2}}\right)^{k_{2}} F_{\omega}\left(z_{1}, z_{2}\right), \quad k_{1}, k_{2} \geqq 0,
$$

are also $K$-linearly dependent $\bmod K\left(z_{1}, z_{2}\right)$. Hence the functions

$$
F^{\left(k_{1}, k_{2}\right)}(z, 1), \quad k_{1}, k_{2} \geqq 0
$$

are $K$-linearly dependent $\bmod K(z)$. We have

$$
F^{\left(k_{1}, k_{2}\right)}(z, 1)=\sum_{h=1}^{\infty} h^{k_{1}}\left\{1+2^{k_{2}}+\cdots+[h \omega]^{k_{2}}\right\} z^{h} .
$$

Put

$$
f_{i j}(z)=\sum_{h=1}^{\infty} h^{i}[h \omega]^{j} z^{h}, \quad i \geqq 0, j \geqq 1 .
$$

Then $\left\{F^{\left(k_{1}, k_{2}\right)}(z, 1)\right\}_{0 \leq k_{1}, k_{2} \leq M}$ and $\left\{f_{i j}(z)\right\}_{0 \leq i \leq M, 1 \leq j \leq M+1}$ generate the same vector space over $K$, and so $\left\{f_{i j}(z)\right\}_{i \geq 0, j z 1}$ are $K$-linearly dependent $\bmod K(z)$. Since the coefficients of $f_{i j}(z)$ are all in $\boldsymbol{Q},\left\{f_{i j}(z)\right\}_{i \geq 0, j \geq 1}$ are $\boldsymbol{Q}$-linearly dependent $\bmod \boldsymbol{Q}(z)$. Then there are integers $e_{i j}$, not all zero, such that 


$$
g(z)=\sum_{i=0} \sum_{j=1} e_{i j} f_{i j}(z)=p(z) / q(z) \in \boldsymbol{Q}(z),
$$

where $p(z)$ and $q(z)$ are relatively prime polynomials with integer coefficients. Let $\xi_{1}, \cdots, \xi_{n}$ be the distinct roots of $q(z)$ and $g(z)=\sum_{n=0}^{\infty} c_{h} z^{h}$. Then we have $c_{h} \in \boldsymbol{Z}$ and

$$
c_{h}=P_{1}(h) \xi_{1}^{h}+\cdots+P_{n}(h) \xi_{n}{ }^{h}, \quad h \gg 0 .
$$

We choose a subset $S$ of $\left\{\xi_{1}, \cdots, \xi_{n}\right\}$ such that for every $i(1 \leqq i \leqq n)$, there exists an unique $\xi \in S$ with $\xi_{i} / \xi$ is a root of unity. We may assume $S=$ $\left\{\xi_{1}, \cdots, \xi_{m}\right\}$. By the choice of $S, \xi_{i} / \xi_{j}$ is not a root of unity for any distinct $i, j$. For a suitable natunrnal number $N$, we have

$$
c_{h N}=Q_{1}(h) \xi_{1}{ }^{h N}+\cdots+Q_{m^{\prime}}(h) \xi_{m^{\prime}}{ }^{h N}, \quad h \geqq 1,
$$

where $Q_{i}(h)$ are nonzero polynomials of $h$ and $m^{\prime} \leqq m$. By (11) and (12), $c_{h}$ are rational integers and

$$
\left|c_{h}\right| \leqq c_{1} h^{c_{2}}, \quad h \geqq 1,
$$

where $c_{1}$ and $c_{2}$ are positive constants. When $m^{\prime} \geqq 1$, by the lemma in [5], we have

$$
\left|\boldsymbol{\xi}_{i}{ }^{\sigma}\right|_{p} \leqq 1, \quad i=1, \cdots, m^{\prime},
$$

where $p$ is $\infty$ or a prime number and $\sigma$ is any automorphism of $\overline{\boldsymbol{Q}}$. Therefore we conclude that $\xi_{i}$ are roots of unity. Hence we have

$$
c_{h N}=a_{8} h^{s}+a_{8-1} h^{s-1}+\cdots+a_{0}, \quad h \geqq 1,
$$

for a suitable natural number $N$. If $m^{\prime}=0$, then $c_{h N}=0$ for any $h \geqq 1$. In any case, we have the equality (13). On the other hand, by (12), we have

$$
\begin{aligned}
c_{h N} & =\sum_{i=0} \sum_{j=1} e_{i j}(h N)^{i}(h N \omega-\{h N \omega\})^{j} \\
& =P_{t}(\{h N \omega\}) h^{t}+P_{t-1}(\{h N \omega\}) h^{t-1}+\cdots+P_{0}(\{h N \omega\}),
\end{aligned}
$$

where $\{x\}$ denotes the fractional part of $x, P_{i}$ are polynomials, $P_{t} \neq 0$ and at least one of $P_{t}, \cdots, P_{0}$ is not constant. Let $t_{0}$ be the largest integer such that $p_{t_{0}}$ is not constant. Comparing (13) and (14), we see that $s=t, a_{i}=P_{i}$ for $i=$ $t_{0}+1, \cdots, t$ and

$$
a_{t_{0}}=\lim _{h \rightarrow \infty} P_{t_{0}}(\{h N \omega\}) .
$$

This is a contradiction, since $\{\{h N \omega\}\}_{h=1}^{\infty}$ is dense in the interval $[0,1)$. 


\section{References}

[1] Kubota, K.K., On the algebraic independence of holomorphic solutions of certain functional equations and their values, Math Ann. 227 (1977), 9-50.

[2] Loxton, J. H. and van der Poorten, A. J., A class of hyper transendental functions, Aequationes Math. 16 (1977), 93-106.

[3] Mahler, K., Arithmetische Eigenschaften der Losungen einer Klasse von Funktionalgleichungen, Math. Ann. 101 (1929), 342-366.

[4] Mahler, K., Arithmetische Eigenschaften einer Klasse transzendental-transzendenter Funktionen. Math. Z. 32 (1930), 545-585.

[5] Nishioka, K., Algebraic independence of certain power series, seminaire de Theorie des Nombres Paris (1987-1988), Progress in Math 81, Birkhäuser, 201-212.

Department of Mathematics

College of Humanities \& Sciences

Nihon University

Sakurajosui, 3-25-40, Setagaya-ku

Tokyo, 156 Japan 